\title{
LUIS ALEJANDRO NOVELO RETANA
}

\author{
(24 DE FEBRERO DE 1951 - 15 DE JUNIO DE 2006)
}

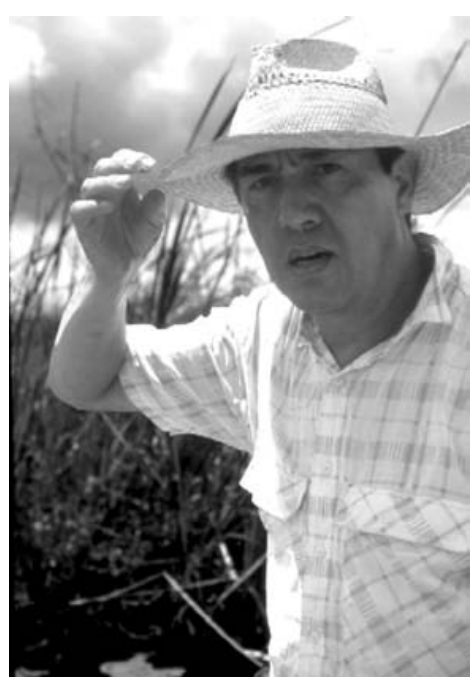

Foto: Carmen Loyola
La muerte no es verdad cuando se ha cumplido bien la obra de la vida

Q uiero compartir la pena que embarga a la comunidad botánica por la pérdida de nuestro querido maestro, colega y amigo, Dr. Luis Alejandro Novelo Retana, investigador del Departamento de Botánica del Instituto de Biología, Universidad Nacional Autónoma de México (UNAM), acaecida el pasado 15 de junio en la ciudad de México. Es el momento para rendir tributo a quien dedicó todo su empeño al desarrollo científico de la botánica acuática de nuestro país, de América y del mundo. Esto lo hizo merecedor de todo nuestro respeto y el público reconocimiento de la comunidad científica en nuestro país y en particular de mi persona.

El Dr. Alejandro Novelo nació en la ciudad de México, Distrito Federal, el 24 de febrero de 1951. Realizó sus estudios de licenciatura en la Facultad de Ciencias de la UNAM, graduándose en 1973, donde también obtuvo su maestría en 1979 y el doctorado en 1996, con la tesis Sistemática de la familia Pontederiaceae en México. Durante los años 1981 a 1982 fue Visitor Fellow en la Universidad de Harvard en Cambridge, Estados Unidos, realizando un programa de especialización académica en las áreas de taxonomía y florística de las familias de plantas acuáticas vasculares, bajo la asesoría del Dr. Richard A. Howard del Arnold Arboretum.

En 1978 recibió una mención honorífica en el certamen de tesis profesionales que se efectuó en el VII Congreso Mexicano de Botánica. En el Sistema Nacional de Investigadores fue Candidato a Investigador Nacional de 1985 a 1988, Investigador Nacional Nivel I de 1988 a 1991 e Investigador Nacional Nivel II de 1991 hasta 2006. Fungió como miembro del Consejo Editorial de la revista Hidrobiológica editada por la Universidad Autónoma Metropolitana-Iztapalapa, miembro del Comité de Línea de Grado del posgrado en Biología Vegetal de la Facultad de Ciencias de la UNAM y miembro del Consejo Técnico Asesor de la Reserva de la Biosfera Pantanos de Centla, Tabasco. Participó además en la Comisión Dictaminadora Divisional de Ciencias Biológicas y de la Salud (UAM-Iztapalapa), fue Secretario Técnico del Instituto de Biología (UNAM) y miembro del Comité Nacional de Evaluación de Métodos para el Control y Aprovechamiento de Malezas Acuáticas.

Impartió clases curriculares de botánica y métodos de estudio de la vegetación acuática, en la Facultad de Ciencias (UNAM) entre 1974 y 1978. En la Universidad Autónoma Metropolitana- Iztapalapa impartió el curso monográfico de vegetales acuáticos de 1978 a 1989. Extracurricularmente, impartió cursos de ecología en la Unidad Profesional Interdisciplinaria de Ingeniería y Ciencias Sociales y Administrativas (Instituto Politécnico Nacional), y en la División de Ciencias Básicas (Biología) de la Universidad Juárez Autónoma de Tabasco, sobre taxonomía, ecología y florística de plantas acuáticas. Más tarde, ofreció el curso limnología aplicada en el Instituto Mexicano de Tecnología del Agua (IMTA) en Morelos, y en la Universidad Autónoma de Sinaloa impartió el primer curso sobre control integrado de la maleza acuática.

Coordinó eventos académicos como el curso Ecología, fisiología y manejo de manglares en la Estación de Biología Tropical Los Tuxtlas, Veracruz, en 1990. Conjuntamente con el Dr. Thomas Philbrick coordinó el Simposio Internacional "Biología de las plantas acuáticas vasculares y su hábitat en Latinoamérica", que se celebró como parte del VII Congreso Latinoamericano de Botánica y XIV Mexicano 
de Botánica en 1998 en la ciudad de México, que lo llevó a tener importantes relaciones académicas con investigadores de algunas partes del mundo.

En el ámbito de la investigación, a partir de 1976 desarrolló proyectos para diversas dependencias de nuestro país, tales como el Instituto Nacional de Investigaciones sobre Recursos Bióticos (INIREB), el Consejo Nacional de Ciencias y Tecnología (CONACYT), Química Hoechst, la Delegación de Xochimilco, la Asociación Amigos de Xochimilco, A. C., Petróleos Mexicanos y el Instituto Mexicano del Petróleo, enfocados principalmente a la ecología de las plantas acuáticas en las regiones tropicales y templadas del país, restauración de humedales, control de malezas acuáticas, la flora acuática de México y Mesoamérica, el inventario de la vegetación acuática vascular de cuatro regiones hidrológicas prioritarias del centro de México y de la Reserva de la Biosfera Pantanos de Centla, Tabasco.

En 1990, le presenté al Dr. Thomas Philbrick (Western Connecticut State University), con quien a partir de 1993 inició conjuntamente proyectos relacionados con la sistemática y evolución de las podostemáceas mexicanas. También desde 1996 ellos colaboraron con la Dra. Judith Márquez de la Facultad de Ciencias (UNAM) en estudios sobre embriología y cariología de las podostemáceas mexicanas, así como con investigadores de Argentina, Brasil, Estados Unidos, Guatemala, Suiza y Venezuela. Alejandro Novelo compartió sus conocimientos con una infinidad de estudiantes e investigadores.

La amplia experiencia en el campo de la botánica acuática del Dr. Alejandro Novelo, le permitió desde el año de 1978 hasta su deceso, publicar numerosos artículos de difusión y científicos en revistas nacionales e internacionales de manera individual y en colaboración con investigadores del país y del extranjero. Sus estudios versan sobre flora y vegetación acuáticas principalmente en los lagos del centro del país o de algunos estados como Tabasco y Tamaulipas, usos de plantas acuáticas en chinampas, nuevos registros de plantas acuáticas en México, como Pontederia rotundifolia (Pontederiaceae), Didiplis diandra (Lythraceae), Myriophyllum quitense (Haloragaceae), Luziola subintegra y L. spruceana (Poaceae), Hydrilla verticillata (Hydrocharitaceae), Nymphaea amazonum (Nymphaeaceae), Spirodela intermedia (Lemnaceae) en México y Costa Rica, Ruppia didyma (Potamogetonaceae) en México y las Antillas, entre otros, y lectotipificó especies como Limnocharis laforestii (Limnocharitaceae).

Interesado en la resolución de problemas taxonómicos, nomenclaturales y sistemáticos de familias de plantas acuáticas, y en colaboración con investigadores nacionales y del extranjero, principalmente con los doctores Thomas Philbrick, Donald Les, Rolf Rutishauser y Judith Márquez, desde 1993 se dedicó a conocer con mayor profundidad la familia Podostemaceae, destacando sus investigaciones en la descripción de nuevos géneros endémicos a México como Vanroyenella, o de especies de Marathrum y Oserya de México, y de Podostemum de México y Brasil. Además, realizó estudios de fitoquímica, biología reproductiva, ecología, evolución, taxonomía, filogenia, citología, botánica estructural, embriología y morfología general, publicando dichos estudios en revistas como Biotropica, Aquatic Botany, Systematic Botany Monographs y Taxon, entre otras.

Entre los años de 1985 a 1990, conjuntamente con el Dr. Antonio Lot, realizó tratamientos taxonómicos de géneros o familias de plantas acuáticas para la Flora Fanerogámica del Valle de México, destacando entre ellos el género Utricularia y las familias Alismataceae, Araceae, Haloragaceae, Hydrocharitaceae, Juncaginaceae, Lemnaceae, Lilaeaceae, Najadaceae, Pontederiaceae, Potamogetonaceae, Typhaceae y Zannichelliaceae. En 1994 ellos dos describieron las familias Alismataceae, Cymodoceaceae, Hydrocharitaceae, Limnocharitaceae, Najadaceae, Pontederiaceae, Potamogetonaceae y Zannichelliaceae para Flora Mesoamericana.

A partir de 1998 describe para la Flora del Bajío y de Regiones Adyacentes con el M. en C. Leandro Ramos, el Dr. Thomas Philbrick y conmigo las familias Mayacaceae, Nymphaeaceae, Podostemaceae y Pontederiaceae, entre otras. Entre los capítulos en

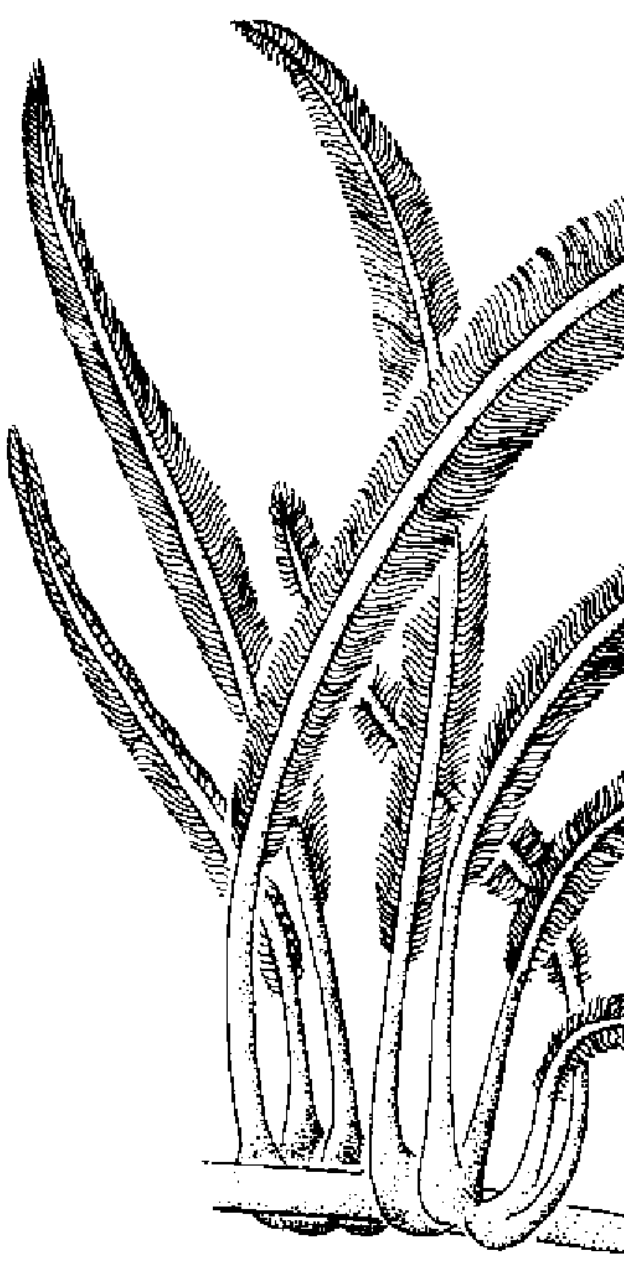




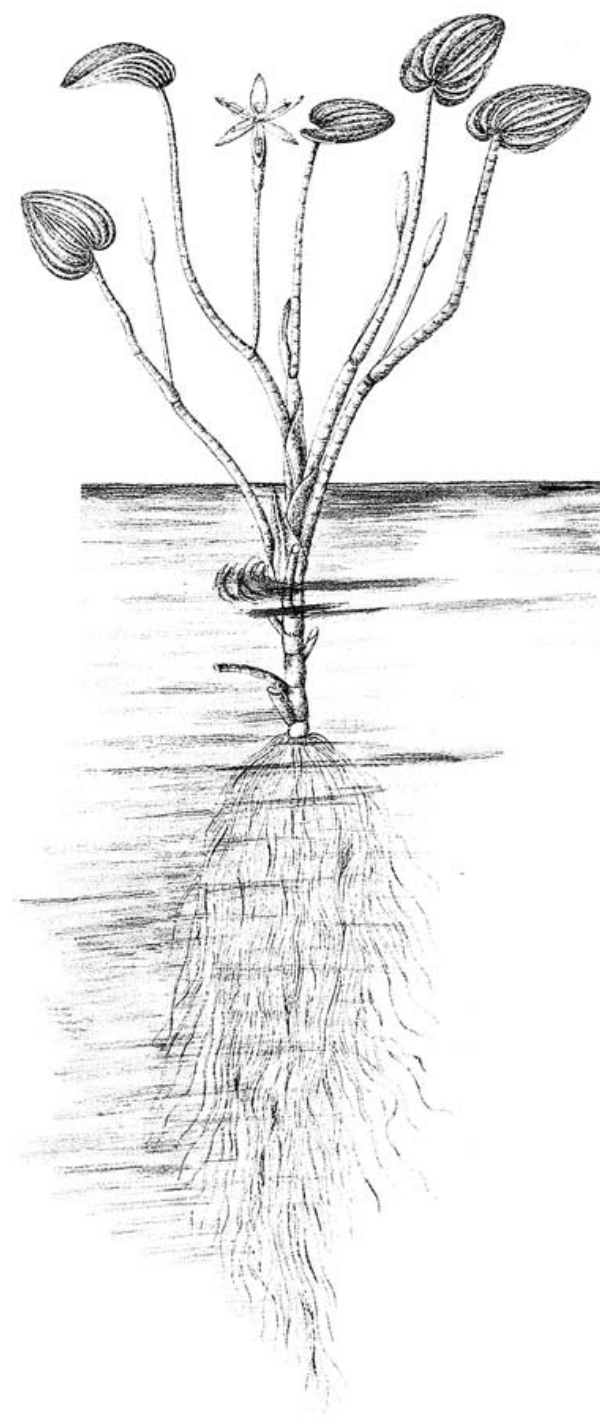

los que participó como coautor de capítulos destacan Forested wetlands of Mexico, Diversity of Mexican aquatic vascular plant flora y Vegetación acuática de Tabasco.

De los libros más importantes de su trayectoria académica en los que él participó conjuntamente con investigadores del Instituto de Biología de la UNAM fueron Angiospermas Acuáticas Mexicanas 1, Catálogo de Angiospermas Acuáticas de México, Hidrófitas Estrictas Emergentes, Sumergidas y Flotantes e Iconografía y Estudio de Plantas Acuáticas de la Ciudad de México y sus Alrededores, este último con excelentes ilustraciones de Elvia Esparza. Entre ambos publicamos el Manual de Identificación de Plantas Acuáticas del Parque Nacional Lagunas de Zempoala, México con fotografías de Carmen Loyola.

Alejandro fue miembro de reconocidas asociaciones académicas tales como la Sociedad Botánica de México, desde 1975, la International Association of Aquatic Vascular Plant Biologist, desde 1982, y The American Society of Plant Taxonomists, desde 1993.

A partir de 1975, sus contribuciones en el campo de la botánica acuática fueron expuestas en diversos foros académicos, desde pláticas o seminarios a grupos de pobladores o de estudiantes, profesores e investigadores en diversas instituciones de educación superior del país, hasta congresos y simposios nacionales e internacionales de reconocido prestigio. Sus temas versaban desde aspectos generales de las plantas acuáticas hasta tópicos como dinámica de poblaciones, flora y clasificación de la vegetación, su importancia, utilización y estado actual, ecología, recolecta e identificación de las plantas acuáticas. La mayoría de estos tópicos los enfocaba a los diferentes ecosistemas acuáticos naturales de nuestro país.

Algo que Alejandro Novelo consideró como muy importante fue expresar sus conocimientos a través de alguien y ésos fueron sus estudiantes con quienes compartió su entusiasmo, amor y pasión por las plantas acuáticas. De ellos personalmente me formó a mí, asesorándome en la licenciatura y dirigiendo mis estudios de maestría y doctorado. Sus estudiantes fueron de diversas instituciones del país tanto de licenciatura, maestría o doctorado. Entre ellos destacan Pedro Ramírez García, Lucía Chávez Heredia, José Rojas Moreno, Leandro Javier Ramos Ventura, Marco Antonio Mijangos Carro, Norma Oropeza Hernández, Adriana García Mendoza, Margarita Elizabeth Gallegos Martínez, Héctor Cáliz de Dios, Guillermina Murguía, Arturo Mora Olivo, Lourdes Cruz Terán, Miguel Ángel Ordóñez Librado, Ociel Mora Martínez, María de Lourdes Carmona Jiménez, Rigoberto Romero García e Ivonne Reyes Ortega. Algunas de estas tesis fueron codirigidas con numerosos colegas de otras dependencias e instituciones.

Quiero resaltar que compartió su sencillez de académico con muchos investigadores del Departamento de Botánica del Instituto de Biología (UNAM), quienes que pudieron conocerlo personalmente y compartir con él jornadas de trabajo y de vida. Por ello, fue muy apreciado. Entre ellos, quienes lean estas líneas sabrán que los estoy mencionando aunque no diga sus nombres porque no acabaría. Alejandro Novelo fue, ante todo, un ejemplo de amigo, sencillo y sin pedir nada a cambio, una persona de una humildad extrema, muchas veces no fácilmente visible. Esta humildad estaba implícita en detener cualquiera de sus tareas por recibir y atender, sin distinción de título o jerarquía, a todos y cada uno de sus ocasionales visitantes, y brindarles sus conocimientos genuinos o un consejo indispensable, a formular un llamado de alerta ético y oportuno, así como a atender con esmero a sus amigos. A todos nos incentivaba.

Su calidad de taxónomo logró motivarme a efectuar estudios detallados de plantas acuáticas. Veinticuatro años de conocernos y de trabajo, dieron por fruto la colección particular más grande de plantas acuáticas de México y ahora en Mesoamérica. Nuestros especímenes colectados están hoy depositados en las principales colecciones botánicas de nuestro país y del extranjero. En algunas ocasiones, no dudaba en desprenderse del espécimen para estimular a aquel joven al que le veía vocación en el campo de la botánica acuática.

Recuerdo que las respuestas que buscábamos en nuestros estudios, estaban allí, en 
el lago, el río, el manantial, la laguna o el mar, y en algunas ocasiones al alcance de las sanguijuelas, de los cocodrilos, de las abejas o de lo helado de los lagos templados.

Juntos comprendimos, ya con una sólida base de estudios taxonómicos, que analizar el grupo de plantas acuáticas era la mejor manera de poder descifrar su taxonomía y biología, e incluso era la forma de acceder a regiones desconocidas, razón por la que iniciamos proyectos florísticos en el centro y sureste del país, así como en Centro y Sudamérica. De este modo, se comenzó a gestar una de las más fructíferas asociaciones de especialistas en este campo de la botánica, y como lo indiqué anteriormente, con decenas de colaboradores, muchos de ellos mis estudiantes a los que Alejandro se sumó en su guía. Su alejamiento de la investigación debido a sus problemas de salud constituyó una durísima prueba en la carrera académica de Alejandro. De su enfermedad nunca pudo recuperarse definitivamente; sin embargo, sus publicaciones del último lustro son un fiel reflejo de su actividad, que nunca cesó.

Además de su pasión por las plantas acuáticas, el deporte fue algo que compartimos. Cuando lo conocí yo había dejado de jugar soccer profesional en tercera división en Morelos, sabiendo él que era lo que más me entusiasmaba, y fue cuando me platicó que al estudiar en la UNAM jugaba voleibol y que había sido seleccionado de esa institución, siendo representante nacional, y que había sido condecorado en el Salón de la Fama. Pero además, Alejandro fue un apasionado de la buena música, de la comida, del canto y de la fotografía. Sus últimos años se dio la oportunidad de disfrutar la naturaleza desde otro punto de vista, a través de la acuarela. Esto lo hizo ser un estudiante de Elvia Esparza, dibujando principalmente las plantas acuáticas como las ninfas mexicanas, tomando como ejemplo las cultivadas en el Jardín de Plantas Acuáticas Xochitla en el Estado de México, proyecto que desarrollé conjuntamente con la M. en C. Lorena Martínez y las Biol. Nayeli González Mateos y Mónica López Hernández y con el entusiasmo del mismo Alejandro Novelo.

Su fallecimiento significa uno de los golpes más duros para mí, que siempre lo seguiré recordando, sin importar los años transcurridos. Sin dejarme abatir, continuaré trabajando intensamente y publicando de igual forma en nombre de él, porque debo concluir proyectos que estábamos desarrollando, porque hay cientos de problemas por resolver y estudiar en el campo de la botánica acuática de nuestro país.

Alejandro Novelo nos dejó físicamente. Ya no lo veremos en su cubículo o por los pasillos del Instituto de Biología y de otras instituciones universitarias. Meses antes de su deceso, planeábamos festejar nuestra "Amistad de Plata" ya que en 2007 cumpliríamos 25 años de una gran hermandad. Sin embargo, antes de partir expresó su última voluntad, la cual cumpliré cabalmente y con ello. Alejandro seguirá viviendo en la academia y en nuestros corazones.

La botánica mexicana pasa por un triste momento y, en especial, la botánica acuática. Con su partida, todos nos hemos tornado piezas descartables. Podemos irnos en soledad y silencio, seguros de que no se vislumbra un reemplazo cercano. Así se fue mi colega y amigo. Rindo dolorido homenaje, a la figura del Dr. Alejandro Novelo Retana.

¡Descansa en paz Alejandro, estás instalado definitivamente en mi recuerdo, en el de tu familia, en el de tus estudiantes y en el de los colaboradores que compartieron contigo una parte de sus vidas!

Jaime Raúl Bonilla Barbosa

Centro de Investigaciones Biológicas

Universidad Autónoma del Estado de Morelos

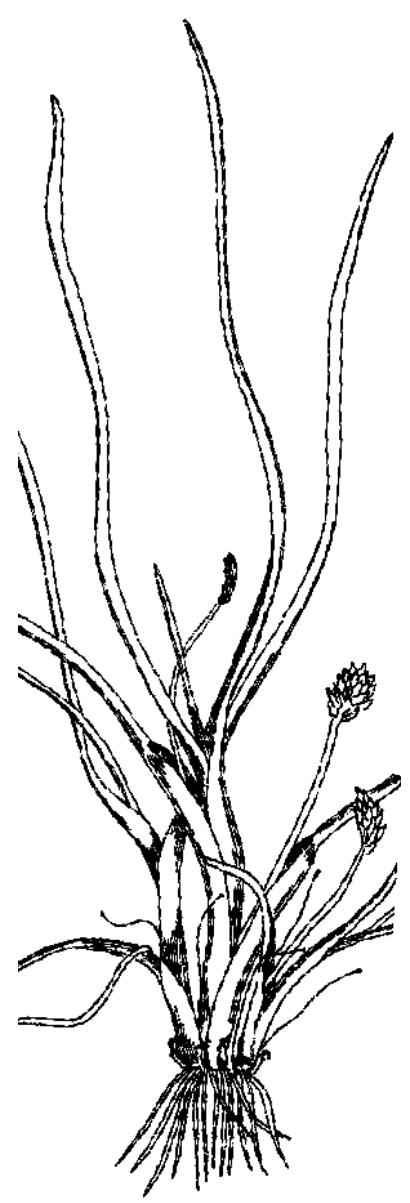

